EPJ Web of Conferences 53, 09006 (2013)

DOI: $10.1051 /$ epjconf/20135309006

(C) Owned by the authors, published by EDP Sciences, 2013

\title{
Pioneering space based detector for study of cosmic rays beyond GZK Limit
}

\author{
B.A. Khrenov ${ }^{1, a}$, M.I. Panasyuk ${ }^{1}$, G.K. Garipov ${ }^{1}$, N.N. Kalmykov ${ }^{1}$, \\ P.A. Klimov ${ }^{1}$, V.S. Morozenko ${ }^{1}$, S.A. Sharakin ${ }^{1}$, A.V. Shirokov ${ }^{1}$, I.V. Yashin ${ }^{1}$, \\ S.V. Biktemerova ${ }^{2}$, A.A. Grinyuk ${ }^{2}$, D.V. Naumov², L.G. Tkachev², \\ A.V. Tkachenko ${ }^{2}$, O.A. Saprykin ${ }^{4}$, A.A. Botvinko ${ }^{3}$, I. Park ${ }^{4}$, J. Lee ${ }^{4}$, G. Na ${ }^{4}$, \\ O. Martinez ${ }^{5}, H$. Salazar ${ }^{5}$ and E. Ponce ${ }^{5}$ \\ ${ }^{1}$ Lomonosov Moscow State University Skobeltsyn Institute of Nuclear Physics (MSU SINP), \\ 1(2), Leninskie gory, GSP-1, Moscow 119234, Russian Federation \\ ${ }^{2}$ Joint Institute for Nuclear Research, Dubna, Moscow Region, Russia \\ ${ }^{3}$ Space Regatta Consortium, Koroliev, Moscow Region, Russia \\ ${ }^{4}$ Physics Department, EWHA Woman University, Seoul, Korea \\ ${ }^{5}$ University of Puebla, Puebla, Puebla, Mexico
}

\begin{abstract}
Space-based detectors for study of extreme energy cosmic rays (EECR) are being prepared as promising new direction of EECR study. Pioneering space device - tracking ultraviolet set up (TUS) is at the last stage of its construction and testing. TUS detector description is presented.
\end{abstract}

\section{INTRODUCTION}

Existing ground-based experimental arrays do not collect statistically confident data on cosmic rays beyond the GZK limit (Extreme Energy Cosmic Rays, EECR). New methods of EECR observation with two orders of magnitudes larger exposure is needed for solution of this problem. Method of EAS fluorescence observation from satellites proposed by Linsley and Benson in 1981 [1] may become such a technique. Russian team (SINP MSU, JINR-Dubna and Space Regatta Consortium) and Universities of Korea and Mexico have prepared space-based detector TUS (Tracking Ultraviolet Setup) for launching on board Lomonosov satellite for testing the new method (Fig. 1).

Space-based detectors have the following advantages:

1. EECR particle track can be observed on a huge area of the atmosphere owing to great distance from the detector to the atmosphere. At orbit height of Lomonosov satellite $(500 \mathrm{~km})$, TUS detector will survey $6400 \mathrm{~km}^{2}$ area of the atmosphere.

2. In one (or more) year of its in-orbit operation, one and the same detector observes the entire celestial sphere. This will allow studying distribution of EECR sources, despite possible inaccuracy of determining primary particle energy. In measurements by ground-based arrays with limited sky coverage variation of event intensity with sky coordinates could be caused by unavoidable difference in measurement of absolute primary particle energy by different arrays.

\footnotetext{
a e-mail: bkhrenov@yandex.ru
}

This is an Open Access article distributed under the terms of the Creative Commons Attribution License 2.0, which permits unrestricted use, distribution, and reproduction in any medium, provided the original work is properly cited. 


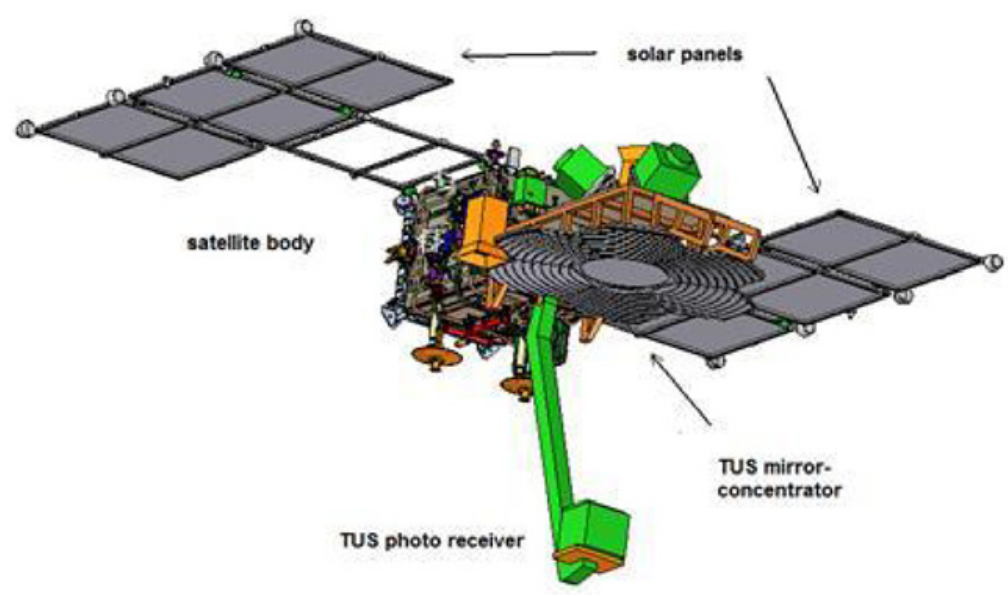

Figure 1. EECR detector TUS on board the Lomonosov satellite.

At the same time, detector on board satellite encounters a number of difficulties:

1. Observation of EAS from a distance approximately 10 times greater than in ground based experiments requires higher sensitivity and resolution of optical system and of photo detector. Desirable resolution of one detector pixel should be equal to diameter of lateral electron distribution in a shower. For satellite orbit height of $500 \mathrm{~km}$, the angular resolution of the orbital detector should be $0.4-2 \mathrm{mrad}$, which is an order of magnitude higher than that of the existing ground-based detectors $(20 \mathrm{mrad})$.

2. Nighttime atmosphere background radiation in UV band (300-400 nm) used for EAS detection varies on satellite route. Data from Universitetsky-Tatiana satellite [2] gave scale of such variation: from $3 \cdot 10^{7}-2 \cdot 10^{8}$ photon $\mathrm{cm}^{-2} \mathrm{sr}^{-1} \mathrm{~s}^{-1}-$ at moonless night (lower value - above oceans, higher value - above aurora zones and cities) to $2 \cdot 10^{9}$ photon $\mathrm{cm}^{-2} \mathrm{sr}^{-1} \mathrm{~s}^{-1}-$ at full moon night. Groundbased arrays operate at moonless nights at specially chosen locations with noise level not more than $5 \cdot 10^{7}$ photon $\mathrm{cm}^{-2} \mathrm{sr}^{-1} \mathrm{~s}^{-1}$.

3. During flight at low latitudes, where electric activity of the atmosphere is high, impulsive noise from lightning and accompanying high altitude discharges will add to average noise level.

4. Technology of an orbital fluorescence detector should satisfy complex conditions of space operation.

Bearing these difficulties in mind a program for gradual conversion of UV fluorescence detector in ground-based version to space version was started [3]. TUS detector is the first, comparatively "simple", instrument that will approve reliability and stability of optical system and photo detector design for operation in space.

\section{SPACE DETECTOR TUS}

Detector consists of two main parts: mirror-concentrator with area of $\sim 1.8 \mathrm{~m}^{2}$ (Fig. 2) and photo detector composed of 256 pixels, located at the mirror focus (Fig. 3). TUS technological parameters are: mass $\sim 60 \mathrm{~kg}$, power consumption $\sim 65 \mathrm{~W}$, data rate 250 Mbytes/day.

Mirror-concentrator is designed as sum of the central parabolic mirror and 11 parabolic rings focusing a parallel beam to one focal point. In this design thickness of the mirror construction is small $(3 \mathrm{~cm})$ which is important for mirror implementation into satellite construction. Mirror focal distance 


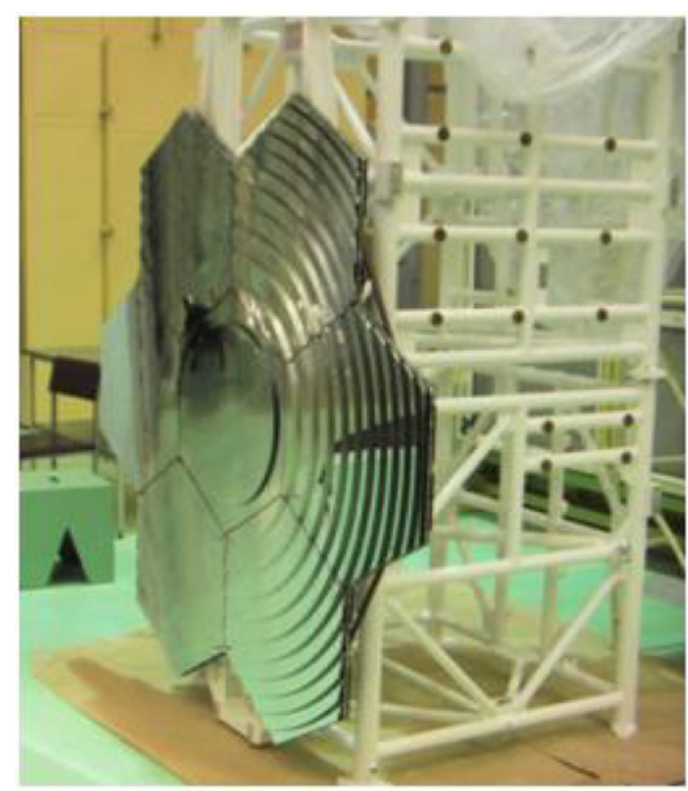

Figure 2. TUS mirror-concentrator on satellite "Lomonosov" scientific payload frame.

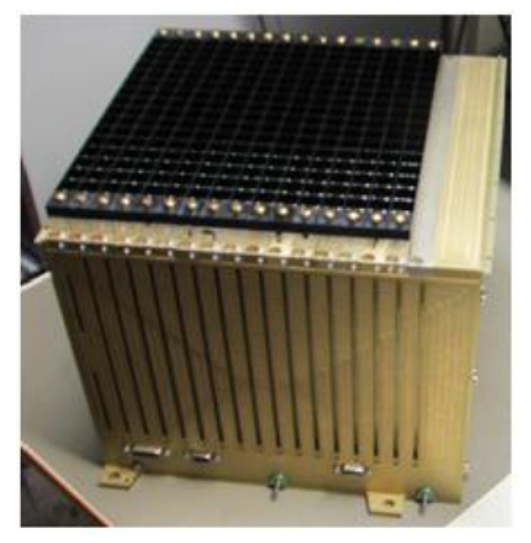

Figure 3. TUS photo detector.

is $1.5 \mathrm{~m}$. The mirror is cut to hexagonal segments with a diagonal $66 \mathrm{~cm}$. Mirror segments are made of carbon plastic strengthened by honeycomb aluminum plate so that the mirror construction is temperature stable in wide range of temperatures. Mirror surface is obtained as plastic replicas of aluminum moulds (one for central mirror part and one for 6 lateral parts).

Plastic mirror surface is covered by aluminum film and protected by $\mathrm{MgF}_{2}$ coat in vacuum evaporation process. Reflectivity of the mirror surface at wavelength $350 \mathrm{~nm}$ (average for the atmosphere fluorescence) is $85 \%$. Expected life time of the mirror is not less than 3 years.

TUS mirror passed various space qualification and optical tests. These tests show stability of optical quality of the mirror in space conditions.

Photo detector pixels are photomultiplier tubes PMT R1463 of Hamamatsu with multi-alcali cathode of $13 \mathrm{~mm}$ diameter. Quantum efficiency of the PMT cathode is $20 \%$ for wavelength $350 \mathrm{~nm}$. PMT's 

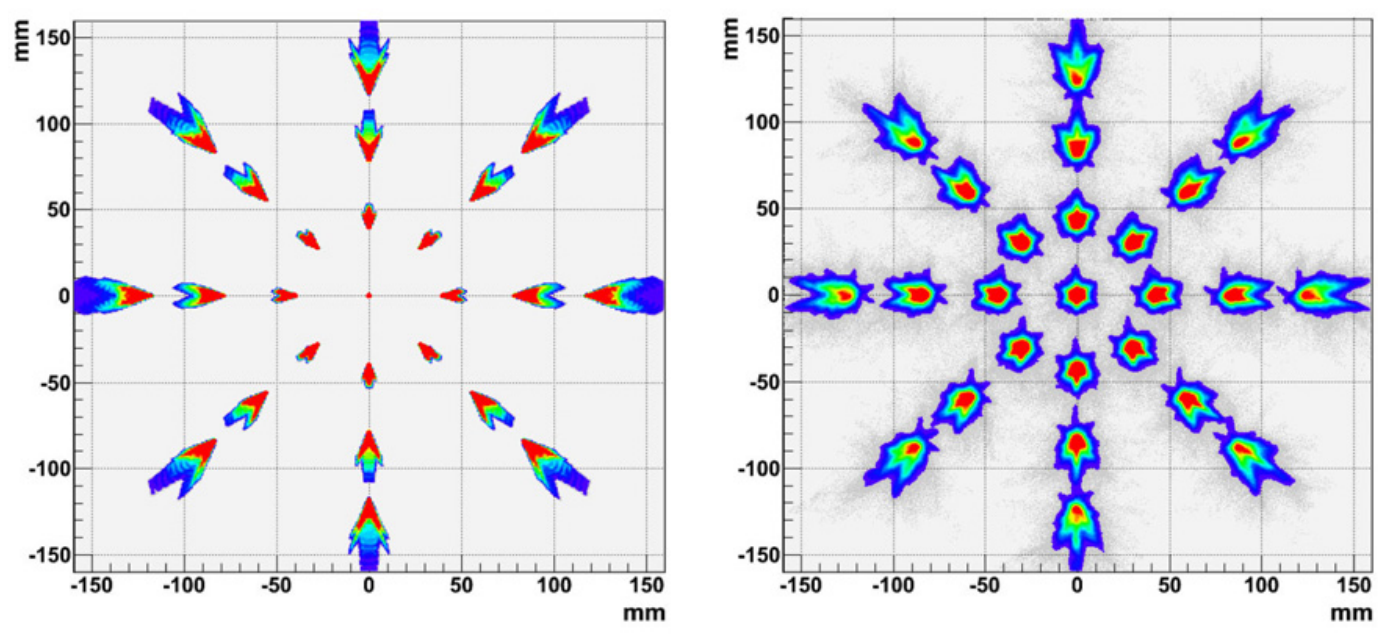

Figure 4. Focal spots (left - "ideal" mirror, right - "real" mirror).

multi-alcali cathode (instead of usually used bi-alcali one in ground-based fluorescence detectors) was chosen for operation in wider range of temperature in which cathode operates in linear regime. To make the detector field of view (FOV) uniformly filled with pixels light guides with square entrance $(15 \times 15 \mathrm{~mm})$ and circle output adjusted to PMT cathode were used.

The qualification testing of phototubes was done by hardware and software complex (test bench) used successfully by JINR group for PMTs test of ATLAS Tile Calorimeter at LHC.

After testing, PMTs with similar gain were grouped in 16 tube clusters. Data from each tube in cluster are digitized by one ADC and then analyzed and memorized by cluster FPGA. Final detector triggering and memorizing of all data is done by central TUS FPGA. Information volume of one EAS data is $\sim 100$ Kbytes. Expected volume of one day EAS data transmitted to the mission center is 250 Mbytes.

\section{DETECTOR PERFORMANCE}

Performance of TUS detector was simulated taking into account parameters of real TUS mirrorconcentrator and parameters of TUS pixels. The main mirror parameter needed for simulation is point spread function (PSF). It was measured in two kinds of control experiment - for every mirror segment with parallel laser beams and for full mirror with point-like source at $30 \mathrm{~m}$ from the mirror. Both results are in agreement and allow to make two-dimensional x, y-coordinate image plot of parallel light beam. Results of PSF measurements are presented in Fig. 4 for "real" mirror (right panel) and "ideal" mirror with requested mirror parameters (left panel). Results are presented for 8 different azimuthal and 4 polar $(\theta)$ angles $\left(\theta=0^{\circ}, 1.5^{\circ}, 3^{\circ}, 4.5^{\circ}\right)$. At small polar angles real mirror images are larger than expected for ideal one. Nevertheless diameter of images is less than pixel size in wide field of view $9^{\circ}$.

Simulations of TUS performance were done with the help of ESAF program designed for JEMEUSO space detector. The main EAS characteristics (energy and direction of primary particle) determine parameters measured by TUS: number of photoelectrons in photo detector pixels as they appear in time. Intermediate parameters: integral number of fluorescent photons in EAS and number of photons coming to detector mirror are in agreements with previous estimates: $\sim 10^{16}$ photons in EAS of energy $10^{20} \mathrm{eV}$ and $\sim 2500$ photons received by TUS mirror.

Photons distribution along EAS "track" in photo detector pixels was simulated taking into account measured mirror PSF. In Fig. 5 percentage of photon number "detected" by TUS pixels is presented 

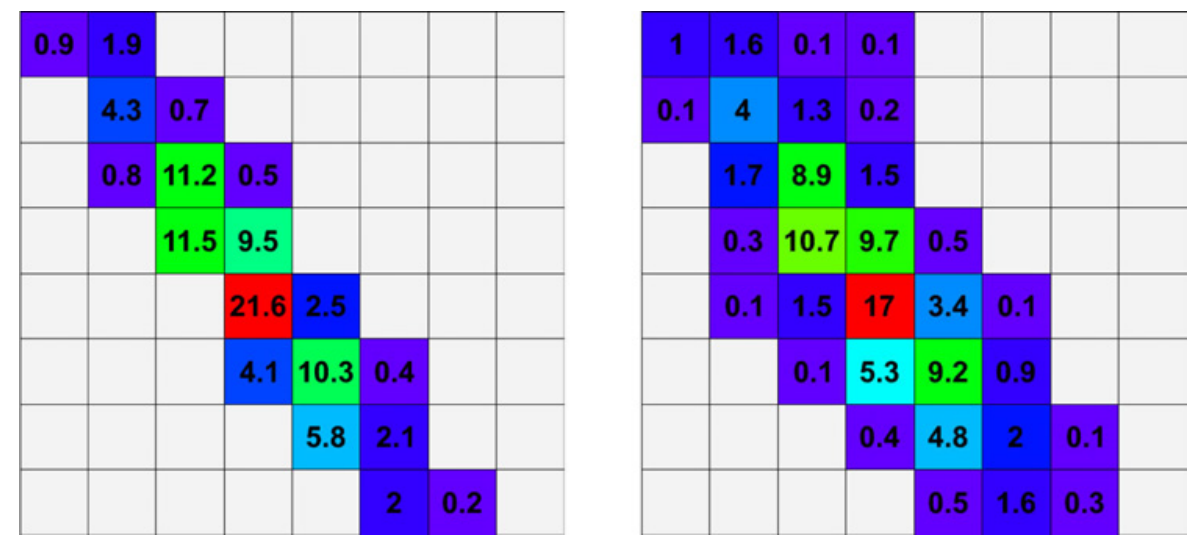

Figure 5. Persentage of EAS photon numbers focused by mirror to one pixel. Left panel: "ideal" mirror, right panel: "real" mirror. Only pixels with percentage more than 0.1 .

for EAS zenith angle $75^{\circ}$. Comparison of photon distribution along EAS pixel track for ideal and real mirrors proves good quality of mirror made for EAS detector with limited number of pixels.

EAS trigger is organized in two stages. In the first trigger stage pixels with signal $A$-times larger than noise RMS are selected. In the second stage event with $B$ neighbor "hit" pixels is recorded as EAS event. Commands with $A$ and $B$ values will be sent from the mission center. They will be selected in compromise between trigger rate (limited by the volume of information transmitted to the mission center every day) and energy threshold of TUS detector. Simulation of triggering is in process. Algorithm for off-line analysis of selected events will be also tested in simulations.

Next space detectors planned to be KLYPVE detector on the Russian segment of ISS [4] and JEMEUSO detector [5] on Japanese experimental module of ISS. TUS detector is a pathfinder for these large scale detectors: it will measure real signal to noise ratio in space observations of EAS.

TUS detector has also other additional tasks: to study transient luminous events (TLE), relativistic dust grains and micro-meteors. These events are developed slower in time, and TUS photo detector electronics should be capable to measure signals in longer temporal scale. As was presented above EECR events with duration of $100 \mu$ s are recorded with temporal resolution of $0.8 \mu$ s in 256 pixels. TLE and relativistic dust grains having duration of millseconds should be recorded in the same number of pixels with resolution of hundreds $\mu$ s. The slowest events (micro-meteors) should be recorded with resolution of tens milliseconds. TUS electronics is designed to satisfy those requests.

\section{References}

[1] Linsley, J., and R. Benson, 17th ICRC Proc. (Paris) 8 (1981) 145

[2] Sadovnichii, V. A., M.I. Panasyuk, I.V. Yashin, et al., Solar System Research. 451 (2011) 3-29

[3] Khrenov, B. A., Panasyuk, M. I., Alexandrov, V. V., et al., AIP Conf. Proc. 566 (2001) 5

[4] Khrenov, B. A., Alexandrov, V. V., Bugrov, D. A., et al., Sov. J. Nucl.Phys (Yad. Fiz.) 67 (2004) 2079

[5] Takahashi, Y. (for JEM-EUSO Collab.), J. Phys.: Conf. Ser. 120 (2008) 062013 